\title{
La Responsabilidad Social Empresarial y las finanzas
}

\author{
Business social responsibility and finance \\ La responsabilité sociale des entreprises et les finances
}

María L. Saavedra García

maluisasaavedra@yahoo.com

Circuito exterior $s / n$, Ciudad Universitaria, Del. Coyoacán, C.P. 04510, México DF - México

Dra. en Administración, Universidad Nacional Autónoma de México. Profesora e investigadora de carrera en la Facultad de Contaduría y Administración de la Universidad Nacional Autónoma de México. Mención Honorífica y Medalla en los estudios de Maestría y Doctorado. Reconocimiento de Investigadora Nacional

del Sistema Nacional de Investigadores (SNI).

Artículo de investigación científica o tecnológica Según Clasificación Colciencias

Fecha de recepción: agosto 01 de 2011 Fecha de corrección: noviembre 26 de 2011 Fecha de aprobación: diciembre 20 de 2011

\section{Resumen}

El objetivo de este artículo consiste en mostrar como la responsabilidad social empresarial (RSE) ha ido cobrando importancia en el mundo de las finanzas. Se realizó a través una revisión documental que permitió desarrollar los temas: El rendimiento económico y la Responsabilidad Social Empresarial, Historia y Evolución de la inversión socialmente responsable, la normatividad acerca de Responsabilidad Social Empresarial en el ámbito financiero y los informes financieros integrados; así como la inclusión del análisis de los casos de Enron de Estados Unidos y Comercial Mexicana de México. Se concluye que la RSE ha ido cada vez cobrando mayor importancia en el ámbito de las finanzas, quedando demostrado esto en que se han creado índices de responsabilidad social, con el fin de que los inversionistas sean capaces de identificar las inversiones socialmente responsables, las cuales se encuentran respaldadas por empresas que han invertido en este rubro. Así también, las empresas han ido adoptando de manera voluntaria la generación y presentación de informes de responsabilidad social empresarial, como complemento de la información financiera.

Palabras clave: Responsabilidad Social, inversión responsable, quiebra de empresas, Enron, Comercial Mexicana. 


\section{Business social responsibility and finance}

La Responsabilidad Social Empresarial y las finanzas

\section{La responsabilité sociale des entreprises et les finances}

\section{La responsabilité sociale des entreprises et les finances}

\author{
La Responsabilidad Social Empresarial y \\ las finanzas
}

\section{Business social responsibility and finance}

\section{Résumée}

Ĺobjectif de cet article est de démontrer comment la responsabilité sociale des entreprises (RSE) acquiert chaque fois plus d'importance dans le monde des finances. On a réalisé une révision documentaire qui nous a permis d'identifier et de développer plusieurs sujets : la rentabilité économique et la responsabilité sociale des entreprises; l'histoire et l'évolution de l'inversion socialement responsable ; la normativité de la responsabilité sociale des entreprises dans le cadre financier, et les rapports financiers intégrés. En plus cette révision a servi pour inclure l'analyse des cas d'Enron aux ÉtatsUnis et de Comercial Mexicana au Méxique.

On a pu conclure que la RSE gagne chaque fois plus d'importance dans le domaine des finances, cela peut être démontré par la création d'indices de responsabilité sociale, grâce auxquels les investisseurs peuvent identifier d'avance les investissements socialement responsables. D’autre part, on a pu constater que les entreprises ont adopté de façon volontaire la présentation de rapports de responsabilité sociale comme complément aux rapports financiers.

Mots clef: Responsabilité sociale, Inversion responsable, banqueroute des entreprises, Enron, Comercial Mexicana. 


\section{La Responsabilidad Social Empresarial y las finanzas}

\section{Introducción}

\section{1. ¿Qué tiene que ver la Responsabilidad Social Empresarial (RSE) con las Finanzas?}

Pareciera ser que el mundo de los valores y el mundo de los números son cuestiones inconexas. Sin embargo, existe hoy en el mundo una tendencia creciente de personas e instituciones que orientan sus políticas de inversión conforme a criterios, no sólo económicos, sino también sociales, ambientales y éticos. Podríamos preguntarnos si esta incorporación de criterios "sociales" es viable.

¿No será, como piensan algunos, que las empresas son más rentables cuando no están atadas a consideraciones "sociales"? ¿Habrá quizás una contraposición entre ética empresarial y rentabilidad? No necesariamente. Pensemos que el uso eficiente de los recursos es en sí un valor ético. De este modo, la rentabilidad de una empresa significa, en primera instancia, que los recursos que se le han confiado se han aplicado con eficiencia y han rendido fruto. Sin embargo, si la mayor rentabilidad de una empresa significara un costo ambiental, social o la participación en un negocio polémico (por ejemplo tabaco, alcohol, armas) es posible pensar que existan costos sociales, ocultos, no evidentes, que impliquen que en última instancia se reduzca 0 , incluso, se anule el beneficio de la empresa para la comunidad. Más aún, con el transcurso del tiempo, estos costos ocultos (o "externalidades", como dirían los economistas) es muy probable que sean "internalizados" mediante una mayor regulación motivada por una creciente toma de conciencia de la sociedad. Lo anterior es congruente con lo señalado por Carroll (1991) sobre la responsabilidad social del negocio que abarca las expectativas económicas, legales, éticas, y discrecionales que la sociedad tiene de las organizaciones en un punto dado en el tiempo.

En este sentido también Núñez (2003) declara que la acción empresarial en la sociedad y en los mercados globales exige alcanzar altos niveles de competitividad, que dentro de un marco de desarrollo sostenible debería ir acompañado de una mayor "solidaridad" y visión ética que permitan atender temas actuales con alto impacto social tales como: protección al medio ambiente, las nuevas tecnologías de información, la innovación y transferencia tecnológica, entre otros.

A principios de los ' 70 , surgieron en los EE.UU fondos "éticos" o "responsables" que no invertían en algunas empresas o industrias cuestionables. Lógicamente querían ganar dinero, pero dentro de ciertas restricciones, coherentes con los principios personales o institucionales de sus fundadores. Lo curioso fue que estos fondos, que al principio eran sólo un fenómeno marginal y anecdótico, crecieron paulatinamente hasta llegar a tener una relevancia innegable. Según estimaciones del Social Investment Forum, hoy en los EE.UU el $10 \%$ de los fondos están invertidos al menos con algún criterio ambiental, social o ético. Al año 2011 gran parte de los principales bancos de inversión del mundo ofrecen al menos algún producto financiero catalogado como ético o responsable (Martínez, 2007).

¿Son rentables estas inversiones? Se han hecho decenas de estudios en el mundo, la tendencia parece ser favorable, aunque todavía no es algo concluyente. Por ejemplo, en un estudio encargado por la autoridad ambiental británica (Environment Agency) en el 2004, el cual a su vez reúne investigaciones previas de varios continentes, se encontraron evidencias a favor de una correlación entre gestión ambiental y resultados económicos. Lamentablemente, hasta donde sabemos, no existen hoy estudios similares en América Latina.

¿Es posible algo así en Latinoamérica? Pensamos que sí: en este sentido resulta muy interesante el ejemplo de la Bolsa de Sao Paulo, Brasil, donde hace muy poco se creó el "Índice de Sustentabilidad Empresaria” (ISE), una herramienta de inversión que consiste en una canasta de acciones de compañías seleccionadas según parámetros sociales y ambientales. Lógicamente, cabe preguntarnos cómo se ha comportado esta canasta de acciones de compañías seleccionadas según parámetros sociales y ambientales. Es decir, cómo se ha comportado esta canasta de empresas respecto al resto del mercado, en otras palabras, si los inversionistas han pagado una prima adicional por tener activos con componentes éticos y ambientales. Tomando los rendimientos desde la creación de ese índice 
(diciembre 2005) hasta agosto del 2010, el mismo ha crecido algo menos que el índice general, aunque parece ser menos volátil que éste. Aún así, el lapso considerado es muy breve como para poder llegar a conclusiones definitivas al respecto (Consejo Profesional de Ciencias Económicas, 2010).

Según la experiencia mundial, un fondo de inversiones que considere estas inquietudes, es visto por el público inversionista con mejores ojos y termina siendo en última instancia atractivo para inversionistas de largo plazo. Desde otro punto de vista, podemos pensar que aquellas empresas que trabajen respetando criterios ambientales y sociales serán prioritariamente elegibles por los fondos éticos que se creen en el futuro. Hay aquí, entonces, una oportunidad para aquellas empresas que tomen la delantera en el camino de la Responsabilidad Social Empresarial.

\section{Metodología}

Este trabajo es una investigación documental que pretende mostrar cómo ha ido cobrando importancia la RSE en el mundo de las finanzas, se utiliza el método histórico para describir la evolución de los aspectos más importantes de este estudio, así como el enfoque descriptivo con el fin de dar a conocer los fenómenos que han logrado el desarrollo cada vez más creciente de la RSE en el ámbito de las finanzas.

\section{El rendimiento económico y la RSE}

Según Drucker (1993) "las empresas, ante la sociedad, tienen la responsabilidad de intentar encontrar soluciones válidas para los problemas sociales básicos que encajen en sus competencias", de otro lado Milton Friedman (1970), Premio Nobel de Economía 1976, argumentaba que una empresa solo tiene "una responsabilidad": los resultados económicos. Conseguir buenos resultados es la "primera responsabilidad", y si no obtiene unos beneficios al menos iguales a sus costos totales, es socialmente irresponsable, pues despilfarra los recursos de los accionistas. Entonces, el rendimiento económico es la base, sin él, la empresa no puede desempeñar ninguna otra responsabilidad; ni puede ser buen empleador, ni generar riqueza, ni ser buen vecino.
Asimismo, Amartya Sen (2003) resalta que los valores éticos de los empresarios y los profesionales de un país, junto con otros actores sociales claves, son parte de sus recursos productivos. Si son a favor de la inversión, la honestidad, el progreso tecnológico y la inclusión social, serán verdaderos activos; si, en cambio, predomina la ganancia fácil y rápida, la corrupción y la falta de escrúpulos, bloquearán el desarrollo. Cuando una empresa es rentable no sólo produce un retorno para sus dueños o accionistas, sino que también genera consecuencias sociales muy deseables, tales como (Gómez, 2007): nuevas oportunidades de empleo a través de su expansión e inversión; provisión de bienes y servicios valiosos para la sociedad; utilidades económicas que el Estado puede gravar y con el producto de esos impuestos mantener a los empleados públicos trabajando, desarrollar la infraestructura, el capital humano, proveer servicios de salud, seguridad social y otros necesarios para mejorar el desarrollo nacional.

Los escándalos empresariales que se han producido durante los últimos años; Enron (2001), WorldCom (2002) o Parmalat (2003), la crisis financiera estadounidense (2007) derivada del uso de la tasa subprime ${ }^{1}$ y de los créditos otorgados a clientes $\mathrm{Ninja}^{2}$, han servido para incrementar la sensibilidad que tienen la sociedad y los mercados sobre las formas que operan y se conducen las empresas. Por consiguiente, la adopción de la Responsabilidad Social Empresarial (RSE), no sólo debe ser una "moda de marketing o de imagen pública", sino que debe reflejar la genuina preocupación de las empresas por el bienestar humano y formar parte de la estrategia corporativa, como lo hacen las más importantes multinacionales, que además, la difunden entre los respectivos grupos de interés nacionales e internacionales.

El argumento de la RSE propone que: una empresa que quiera ser competitiva en el futuro, no sólo puede pensar en conseguir beneficios, sino que además tendrá que hacer compatible su labor productiva con una política que actúe en beneficio del entorno en el que desarrolla su actividad, basándose en principios como el respeto a los derechos humanos, la mejora de las relaciones con la sociedad que le rodea y el respeto al medio ambiente.

1. La tasa subprime es una tasa por encima del promedio y se ofrecen a personas que cuentan con un historial crediticio limitado por lo que son considerados de riesgo elevado.

2. Un cliente Ninja es aquel que no dispone de ingresos fijos, empleo fijo, o propiedades, ver: Saavedra, M. (2008). 
La mayor parte de las firmas que componen el Fortune 500, al año 2010, describen en su página web, los logros en materia de RSE y casi la mitad de las incluidas, cuentan con informes específicos sobre aspectos sociales y, medioambientales de sus actividades. También se ha comprobado que las diez empresas más admiradas del mundo, según el ranking de Pricewaterhouse Coopers, desarrollan prácticas de la RSE y las difunden para aprovechar los efectos positivos que genera la transparencia informativa.

Aún más, se abre paso la tendencia hacia un modelo de empresa, que de acuerdo con el "código de buen gobierno de la empresa sostenible3", que se caracteriza porque crea valor económico, medioambiental y social a corto y largo plazo, contribuyen de esta forma al aumento del bienestar de las generaciones presentes y futuras.

Definitivamente, las empresas que tienen un comportamiento socialmente responsable, diseñan sus estrategias y establecen sus procedimientos internos de gestión teniendo en cuenta no sólo la dimensión económica de sus acciones, sino también la social y la medioambiental. Es decir, la RSE engloba todas las decisiones empresariales que son adoptadas por razones que a "primera vista" se encuentran más allá de los intereses económicos, financieros y técnicos de las empresas.

Las finanzas, en el contexto de la RSE se materializan de forma destacada en los Estados Unidos, donde es creciente la presión pública y ha surgido el intento de crear, junto a los indicadores de calidad usuales, un ISO de calidad social que permita a los "inversionistas" elegir empresas que la practiquen. Las firmas con estrategias claras y desarrolladas con responsabilidad social están siendo "mejor" valoradas por los mercados y además, los más prestigiosos fondos de inversión americanos ya no invierten en empresas que no dispongan de memoria social y medioambiental.

Sin duda, estas actuaciones representan un factor competitivo, pues estas compañías se encuentran en mejor disposición para los analistas e inversionistas bursátiles.

Los índices especializados como Dow Jones for sustainability o el FTS4 Good, han evolucionado mejor que los índices bursátiles tradicionales. Y, además, prestigiosos fondos de inversión americanos, no invierten en firmas que no dispongan de memoria social o medioambiental.

En Europa, existían al año 2001, 280 fondos éticos o ecológicos, con un crecimiento del $76 \%$ respecto a 1999. Y en el año 2007 , el número de estos fondos ascendía ya a 437, siendo el monto de activos invertidos en estos fondos 48,735 millones de euros (Ferruz, San Juan y Muñoz, 2009). Los países que lideran este campo son Bélgica, Suecia, Inglaterra y Francia. Siendo este último el país que más dinamismo está demostrando en los últimos años. De manera especial, pide que se invierta en empresas que se destaquen en valores como los derechos humanos, el respeto y el desarrollo de las personas, y que realicen inversiones "constructivas" en países en desarrollo (Bolsa de Madrid, 2004).

Para España, el punto de partida fue el año 1997, cuando se lanza el primer fondo que invertía exclusivamente en el "sector medioambiental". Posteriormente, en 1999 se crearon los dos primeros "fondos éticos. En comparación con otros países europeos, el crecimiento ha sido más lento y se encuentra en esta creciente tesitura de ofrecer a los inversionistas activos financieros socialmente responsables.

Estos son los "fondos ecológicos", que invierten en empresas que ayudan a mejorar las condiciones medioambientales; y los "éticos"; que canalizan sus inversiones hacia compañías que se comprometen a mejorar la calidad de vida a través de actividades y negocios como la sanidad, la educación o el ocio, excluyendo realizar inversiones en sectores como el armamento, alcohol, tabaco, juego y pornografía. De tal manera, que se comprueba una acelerada muestra de interés en el tejido empresarial español, destacándose los grandes bancos y empresas que precisamente se encuentran establecidas en Latinoamérica principalmente Argentina, en donde a través de una encuesta se detectó que el $86.5 \%$ de los consumidores manifiesta que la responsabilidad social es la que define sus compras; el $52.6 \%$ está dispuesto a pagar más por el precio de los productos de empresas "socialmente responsables" y el $77 \%$ a dejar de comprar los productos y servicios de las irresponsables. 
La inversión socialmente responsable viene creciendo a tasas cada vez mayores por lo que se han creado índices para estas inversiones desde 1988. A continuación se presentan los principales índices y los criterios que involucran (Cuadro 1 ).

\section{Cuadro 1. Índices de inversiones socialmente responsables}

\begin{tabular}{|c|c|c|}
\hline Índice & $\begin{array}{l}\text { Lanzamiento } \\
\text { al mercado }\end{array}$ & Criterios \\
\hline $\begin{array}{l}\text { KLD Global Sustainability } \\
\text { (Inglaterra) }\end{array}$ & 1988 & Medioambiental -Social -Gobierno Corporativo \\
\hline $\begin{array}{l}\text { Ethibel Sustainability Index } \\
\text { (Bélgica) }\end{array}$ & 1992 & $\begin{array}{l}\text { Política social interna - Política medioambiental } \\
\text { Política social externa - Política ética-económica }\end{array}$ \\
\hline $\begin{array}{l}\text { DJSI - Dow Jones Sustainability Indexes } \\
\text { (Estados Unidos) }\end{array}$ & 1999 & Económica - Medioambiental - Social \\
\hline $\begin{array}{l}\text { FTSE4 Good - Financial times stock } \\
\text { Exchange for good index series. (Inglaterra) }\end{array}$ & 2001 & $\begin{array}{l}\text { Oposición al soborno - Derechos humanos } \\
\text { Social y del stakeholders - Medioambiental } \\
\text { Estándares de cadena de suministro laboral }\end{array}$ \\
\hline $\begin{array}{l}\text { ISE - Índice de sustentabilidad empresarial } \\
\text { de bolsa de Brasil }\end{array}$ & 2005 & Económica - Financiera - Social - Medioambiental \\
\hline Índice de sustentabilidad de la BMV México & 2011 & Medio ambiente - Social - Gobierno Corporativo \\
\hline
\end{tabular}

En México, la Bolsa Mexicana de Valores eligió a la empresa inglesa EIRIS (Experts in Responsible Investments) para desarrollar la metodología de evaluación. EIRIS tiene más de 26 años de experiencia en el análisis ambiental, social y de gobierno corporativo de las empresas, siendo la proveedora de información de una serie de índices de sustentabilidad de la Bolsa de Valores Inglesa FTSE -los índices FTSEE4Good-.

La metodología se basa en el análisis de la información pública de las empresas en las áreas de:

- Medio ambiente: políticas, sistemas y reportes, cambio climático, biodiversidad, análisis del producto, etc.

- Social: empleados, comunidad, clientes y proveedores, derechos humanos, no discriminación, salud y seguridad en el trabajo, relaciones con sindicatos, entre otros.

- Gobierno Corporativo "governance" estructura y funciones del consejo, ética, lucha contra la corrupción, etc.

Los beneficios de implementar este tipo de índices, se pueden ver en mercados como el de Brasil, que desde que desarrolló su índice de sustentabilidad hace cinco años, se han visto beneficiados por la Inversión Socialmente Responsable (SRI) que cada año aumenta en valor. Se considera que por lo menos el $10 \%$ de la inversión global es ya inversión socialmente responsable, lo que se traduce en un monto de casi tres billones de dólares en el mundo.

Sin embargo, Kramer y Porter (2006), han señalado que los criterios utilizados en los índices varían ampliamente. Así por ejemplo, el Dow Jones Sustainability Index, incluye aspectos de desempeño económico en su evaluación. Pondera el servicio al cliente casi $50 \%$ más que la ciudadanía corporativa. En contraste, el igualmente prominente FTSE4Good Index no contiene en absoluto indicadores de desempeño económico o servicio al cliente. Incluso, cuando los criterios son los mismos, invariablemente se ponderan en forma distinta en el puntaje final. Con lo anterior podemos ver que los índices no son comparables y se debe tomar en cuenta este aspecto al momento de intentar interpretar los mismos, así como el hecho de que su principal debilidad radica en que los datos son reportados por las propias empresas sin verificación externa.

A pesar de las debilidades que puedan presentarse, se considera que el índice de 
sustentabilidad es un parámetro idóneo para reconocer las mejores prácticas de sustentabilidad de las empresas con el objetivo de construir una economía sólida y adecuada al contexto actual y futuro. De este modo dicho índice permite:

a. Reconocer empresas para la inversión responsable

b. Ser una herramienta para la investigación sobre empresas responsables.

c. Ser un marco de referencia y comparación con otras empresas.

d. Identificar áreas de oportunidad en cuestiones de sustentabilidad

e. Incentivar a las emisoras a comprometerse con la sustentabilidad

La relación entre la RSE y el rendimiento económico, aun no se encuentra definida aunque se han realizado numerosos estudios al respecto, así tenemos (Cuadro 2).
Como podemos ver en el cuadro anterior, aunque no existe un consenso en los resultados de los estudios realizados, sin embargo, son más numerosos aquellos que han comprobado que existe una relación positiva entre la RSE y la Rentabilidad.

\section{Historia y evolución de la inversión socialmente responsable}

La inversión socialmente responsable es aquella que a los criterios financieros tradicionales incorpora criterios sociales y medioambientales. Esta filosofía de inversión permite a los ahorradores ser congruentes con su modo de pensar y pueden servir, incluso, como mecanismo de presión desde el sector privado para cambiar, 0 al menos orientar, las decisiones empresariales de acuerdo a aspectos sociales o medioambientales. Además, de estas inversiones se benefician un conjunto de pequeños proyectos y microempresas que no pueden obtener fácilmente financiamiento al no ofrecer las garantías convencionales que exigen las entidades financieras tradicionales.

\section{Cuadro 2. Relación entre RSE y Rendimiento Económico}

\begin{tabular}{|c|c|}
\hline $\begin{array}{l}\text { Relación entre } \\
\text { RSE y rendimiento } \\
\text { económico }\end{array}$ & Autor/año \\
\hline
\end{tabular}

Positiva

Ninguna

Negativa
Bragdon y Marlin (1972); Moskowitz (1972); Browmna y Haire (1975); Parket y Eilbirt (1975); Belkaoui (1976); Fry y Hock (1976); Sturdivant y Ginter (1977); Bowman (1978); Chugh, Haneman y Mahapatra (1978); Preston (1978); Spicer (1978a); Anderson y Frankle (1980); Shane y Spicer (1983); Rockness, Schlachter y Rockness (1986); Cotril (1990); Fombrun y Shanley (1990); Erfle y Fratantuono (1992); Roberts (1992); Thompson, Smith y Hood (1993); Barth y MacNichols (1994); Cohen, Fenn y Naimon (1995); Diltz (1995); Hart y Ahuja (1996); Klassen y McLaughlin (1996); Nehrt (1996); Pava y Krausz (1996); White (1996); Blacconiere y Northcurt (1997); Kotey y Meredith (1997); Russo y Fouts (1997), Waddock y Graves (1997); Ahmed, Montagno y Firenze (1998), Edwards (1998); Karake (1998); Stanwick y Stanwick (1998); Butz y Plattner (1999); Carter, Kale y Grimm (2000); Blank y Carty (2001); Webley y More (2003); Little y Business in comunity (2003); Garz, Volk y Gilles (2003); Mengue y Ozanne, Morgan Stanley's y Oekom Research (2003); Becchetti, Di Giacomo y Pinnacchio (2008); Becchetti y Ciciretti (2009)

Floger y Nutt (1975); Alexander y Buchholz (1978); Ingram (1978); Abbott y Monsen (1979); Chen y Mercalf (1980); Jedia y Kuntz (1981); Freedman y Jagi (1982); Cochran y Wood (1984); Aupperle, Carroll y Hatfield (1985); Sturdivant, Ginter y Sawyer (1985); McGuire, Sundgren y Schneeweis (1988); Patten (1990); Balabanis, Phillips y Lyall (1998); Berman, Wicks, Koha y Jones (1999); Kreander (2000); Mc Williams y Siegel (2000); Wagner, Van Phu, Azomahou y Wehrmeyer (2002)

Vance (1975); Spicer (1978b); Trotman y Bradley (1981); Ingram y Frazier (1983); Mahapatra (1984); White (1991); Jaggi y Freedman (1992); Hamilton (1995); Feldman, Soyka y Ameer (1996); Cordeiro y Sarkis (1997); Cormier y Magnan (1997); Konar y Cohen (1997); Tomas y Tonks (1999) 
Hoy en día la forma más extendida de invertir con criterios de responsabilidad social es a través de la participación en fondos de inversión o de pensiones éticos o socialmente responsables estos fondos permiten dirigir el ahorro de forma colectiva hacia determinados valores que, además de ser rentables, conllevan la financiación de empresas socialmente responsables, aunque no siempre existieron. A continuación se presenta en el cuadro 3, la historia y evolución de la inversión socialmente responsable (Cuadro 3 ).

Como podemos observar la inversión ética tradicional ha tenido una motivación básicamente moral ideológica o social y se ha centrado en el boicot o la exclusión de determinados sectores industriales de las carteras de inversión. Sin embargo, la nueva corriente que se va imponiendo en los últimos años y que podríamos denominar inversión sostenible, desde una visión más financiera y con perspectiva a largo plazo, trata de incorporar de forma integrada criterios económicos, medioambientales y sociales en la evaluación tradicional de las carteras financieras invirtiendo en las mejores empresas de cada sector.

Respecto de América Latina y el Caribe, la información disponible acerca de la implementación de la RSE todavía no es suficiente. Al igual que en otras regiones del mundo, hace falta el desarrollo de indicadores de desempeño en RSE, que permitan tener un estándar para la medición del desempeño social y ambiental de las compañías, y de los beneficios para el negocio derivados de las prácticas responsables (Flores, et al., 2007). Con excepción del Banco Nacional de Desarrollo de Brasil (BNDES), los bancos y agencias gubernamentales sólo han logrado

\section{Cuadro 3. Historia y evolución de la inversión socialmente responsable}

\section{Año Descripción}

1950 Grupos religiosos cuáqueros, decidieron comenzar a discriminar sus políticas de inversión y dejar de invertir en aquellas actividades (tabaco, alcohol, pornografía y juego) que, de acuerdo a sus idearios, dañaban el tejido moral de la sociedad.

1970 Grupos activistas contrarios a la guerra de Vietnam, se dieron cuenta de que la inversión podría ser un importante instrumento de presión ante las empresas, y se podrían introducir estímulos al cambio de las mismas, ya que su dinero estaba contribuyendo a financiar las empresas que suministraban armamento.

1980 En Francia e Inglaterra se toman iniciativas del tipo boicot contra aquellas empresas que trabajaban en Sudáfrica durante el régimen del Apartheid. Los grupos evitaban financiar indirectamente actividades o regímenes que rechazaban.

1997 Aparece el estándar voluntario (Social Accountability) emitido por CEPAA (Council economic priorities acreditation agency), su objetivo es asegurar una producción ética de bienes y servicios, estableciendo para ello una serie de códigos básicos en relación con los derechos humanos y las condiciones de trabajo a lo largo de toda la cadena de valor. Adicionalmente, para llevar a cabo este proceso, la organización debe introducir un sistema de gestión social elaborado por la norma de calidad ISO 9000, que asegure la mejora continua en los aspectos antes citados.

2000 A principios de este año, los inversionistas tradicionales empiezan a considerar que las buenas prácticas acreditadas en materia de RSE son un buen indicador de la calidad de la gestión y el gobierno de una determinada empresa, apoyado por un marco regulador en países referentes.

En julio de 2000 se modificó la legislación británica sobre fondos y planes de pensiones de 1995, conocida como Disclosure Act, resultando clave para el desarrollo de RSE, ya que exigían transparencia informativa de los gestores de estos fondos, y en, concreto, pedía informar cómo los factores sociales y medioambientales eran tomados en cuenta al momento de decidir invertir o desinvertir en valores cotizados. Esta medida fue replicada en Alemania y Bélgica (2001) y posteriormente en Australia (2003).

2003 Una mayoría de fondos de pensiones británicos han incorporado consideraciones de RSE en sus decisiones de inversión, y el $90 \%$ de los gestores de fondos valoran la RSE como fundamental para el capital reputacional y de marca de las compañías. Se trata de grandes inversionistas institucionales, como Hermes/British Telecom, que gestiona 4 de los 7 mayores fondos de Inglaterra. Asimismo también se han asimilado a este concepto las compañías aseguradoras británicas, entre las que destaca Association of British Insurers (ABI). 
la incorporación general de procedimientos de transparencia, que además, son de carácter obligatorio de acuerdo a los tratados internacionales (Corporación Andina de Fomento, 2004).

\section{La normatividad acerca de RSE en el ámbito financiero}

El financiero es uno de los sectores de actividad pioneros en cuanto a la incorporación de políticas y prácticas de RSE orientadas a la sostenibilidad. Por su influencia en el conjunto del sistema económico y el acervo acumulado de experiencias y acciones en esta materia, el sector financiero es uno de los más interesantes para su estudio. A continuación se presenta en el cuadro 4, la normatividad en el ámbito de la responsabilidad social empresarial, que compete a este sector (cuadro 4).

En definitiva, el sector financiero destaca, respecto a otros sectores, por su amplia presencia en las iniciativas que configuran el esquema de la RSE, pero también por el desarrollo de aproximaciones a los objetivos de esas iniciativas basadas en las especificidades de su negocio.

Respecto de Latinoamérica, aunque sería posible mostrar el progreso en casos como el Acta Ambiental Brasilera y el papel que ha asumido activamente el gobierno argentino en distintos niveles, en términos generales la mayoría de los gobiernos ha hecho esfuerzos muy marginales para integrar consideraciones ambientales (Corporación Andina de Fomento, 2004).

\section{Informes financieros integrados. Tendencia mundial}

Cada vez son más las empresas que integran su informe anual y de contabilidad con el de sostenibilidad. Dicha integración se deriva del cada vez mayor grado de conciencia que hay entre las compañías sobre el valor y la necesidad de presentar un informe que reporte su impacto ambiental y social junto con el desempeño financiero. Recientes acontecimientos que han tenido lugar en varias empresas, así como opiniones de las 20 compañías más grandes de Europa, confirman que las organizaciones han optado por la presentación de informes integrados, según lo muestra un estudio de KPMG (2010): los informes convergen como resultado del giro que ha dado la responsabilidad de los negocios, las memorias de RSE han aumentado de forma significativa en un lapso de diez años desde el 35 por ciento en 1999 al 80 por ciento de las sociedades cotizadas incluidas en la lista Global Fortune 250 en 2008. En el caso de Brasil, la cantidad de informes sociales y de sostenibilidad que presentan las empresas (tanto multinacionales como nacionales) es cada vez mayor, llegando a 500 en el año 2002 (Núñez, 2003). En Argentina, se ha intensificado la actividad de algunas asociaciones empresariales que buscan que se difunda de manera integral el concepto de RSE en la gestión empresarial. Aunque en México aún no se está dando esta tendencia, es un hecho que las empresas más importantes están marcando la pauta y tarde o temprano muchas otras seguirán su ejemplo. La reflexión de ser sustentable a lo largo del tiempo ha llegado a formar parte de los temas a tratar por el Consejo de Administración y, más importante aún, se ha vuelto una estrategia de la compañía, por lo que resulta lógico que ahora también se revele el valor agregado que representa la sustentabilidad en el informe anual.

Con todo, la integración de los informes debe cumplir con varias condiciones y contar con el respaldo de una incorporación real y profunda de los procesos de la administración, incluyendo la dirección y los controles para ser considerada creíble, confiable y sólida que responde a las necesidades de información de los grupos de interés. Hay quienes proponen, incluso, que estos informes presenten en forma anual un Informe Cuantitativo a través de valores absolutos y de indicadores. Lo que habitualmente se difunde como compendio de objetivos y resultados no es un balance al estilo tradicional. Es una memoria, un detalle de hechos -en general no cuantificados ni con objetivos medidos y comunicados- de esfuerzos en el campo social. Un Informe Cuantitativo refleja resultados y permitiría evaluar desempeño en términos de activos y pasivos durante un periodo (Rodríguez, 2007).

Perera, socio de Pricewaterhouse Coopers en Chile (Vives y Peinado, 2004), es uno de los tantos inconformes por la falta de un formato uniforme para los distintos instrumentos utilizados en la medición y notificación de los resultados de la responsabilidad social de las empresas (véase la figura 1). Estos instrumentos pueden agruparse en tres tipos de normas: de contenido, de presentación y de verificación. La propuesta consiste en establecer una relación entre las cifras financieras y las dimensiones sociales 


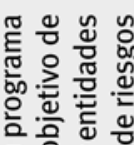

与웡워

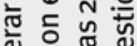

讨

匹

吅 和

을 는 는

융을 융

ปั

은 돈

क जั ํํำ

ธิن

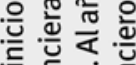

ข 동

荧

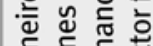

즈 은 눈

옹 형 웜

政

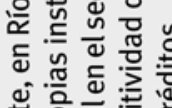

흔듄

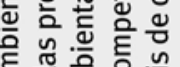

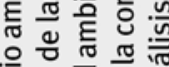

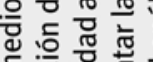

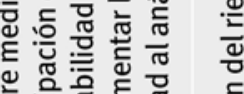

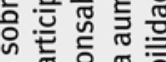

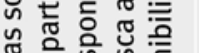

은 뜬 ⿹ㅚㅁ

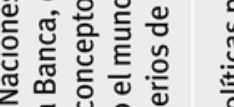

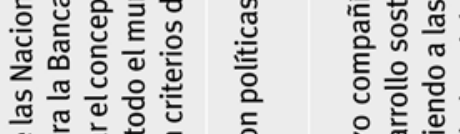

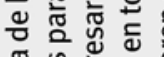

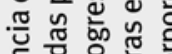

뒁음 은 눙

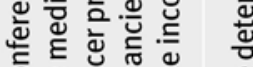

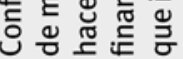

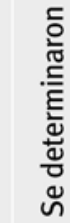

든 응 은

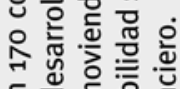

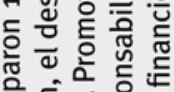

递完高

壳

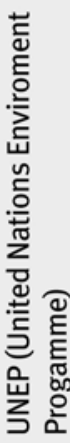

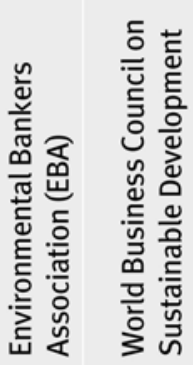

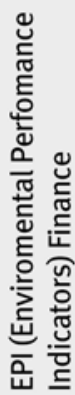

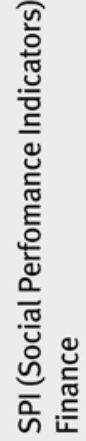

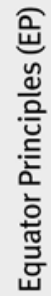

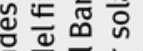

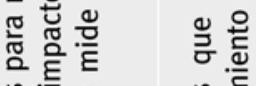

휴웧ㅁ

을 은

들

웜

品 ه

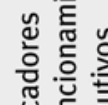

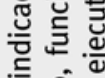

흔원 흔

品

ह

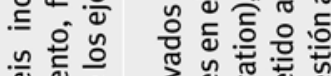

品. 을

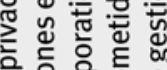

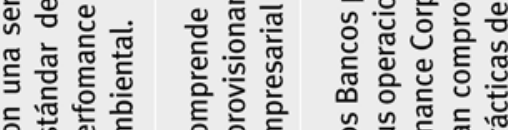

대응 ⿺辶

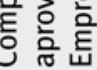

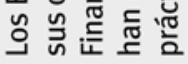

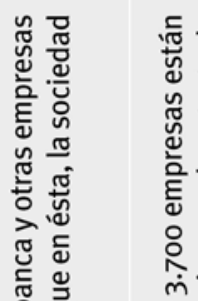

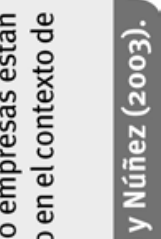

인

ㄸำ ปै

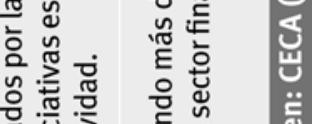

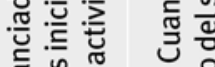

这

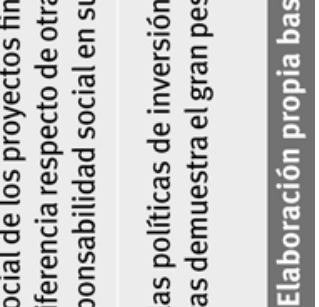

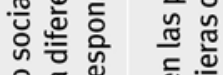

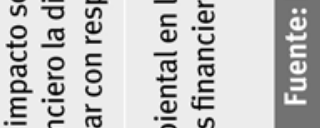

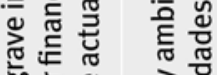

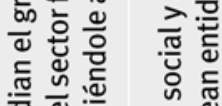

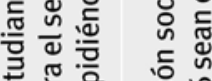

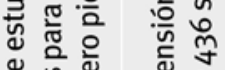

施

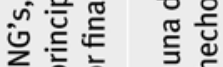

응 형

웡 원

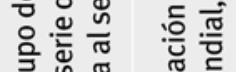

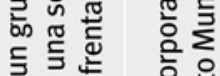

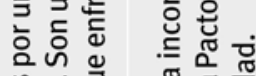

กั

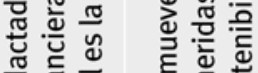

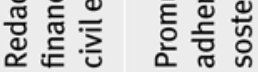

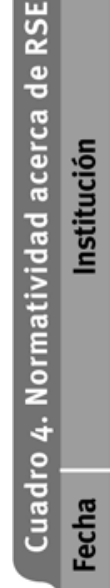

ลั

๙ั ஃ

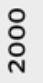

กั่

$\stackrel{m}{\stackrel{n}{\circ}}$

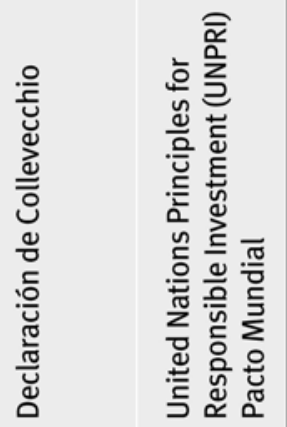


de las empresas en un formato único, a saber, un "cuarto estado financiero" práctico y fácil de analizar. A través del cual se notifica en qué proporción se distribuye el valor generado por una empresa entre los distintos grupos, y a la vez, se complementa la información contenida en los otros tres informes: estado de pérdidas y ganancias, balance general y flujo de caja. Este cuarto formato uniforme permitirá establecer comparaciones período a período, empresa a empresa, e industria a industria (Figura 1).

\section{Figura 1. Informes de responsabilidad social}

\begin{tabular}{|l|c|c|}
\hline Código (GC.OCDE) & $\begin{array}{c}\text { Estándares de } \\
\text { Gobernabilidad } \\
\text { Corporativa }\end{array}$ & $\begin{array}{c}\text { Sistemas de Gestión } \\
\text { (ISO) }\end{array}$ \\
\hline $\begin{array}{c}\text { Estándares de } \\
\text { Desempeño }\end{array}$ & $\begin{array}{c}\text { Reportar el } \\
\text { desempeño (GRI) }\end{array}$ & $\begin{array}{c}\text { Estandares de } \\
\text { Verificación } \\
\text { (AA1000AS, I SAE } \\
3000)\end{array}$ \\
\hline
\end{tabular}

Fuente: Deloitte (2011).

La primera parte del cuarto estado financiero da cuenta de la riqueza que genera una empresa: sus ventas menos sus costos directos 0 , en otras palabras, el valor agregado generado. La segunda parte describe la distribución de la riqueza producida: cuánto se destina a cada uno de los principales grupos de interés (i.e. empleados, estado, comunidad, accionistas y la empresa a través de reinversión). Ésta va acompañada de una serie de notas que permitan contextualizar y entender las cifras de generación y distribución de riqueza. Las notas mínimas incluyen los siguientes anexos en el reporte de cifras básicas:

* Políticas de responsabilidad social:

- Políticas relacionadas con la gobernabilidad corporativa

\section{- Códigos de ética}

- Medio ambiente, seguridad y salud en el trabajo

- Inversión social y desarrollo de la comunidad donaciones, voluntariado y filantropía

- Mercadeo responsable y protección del consumidor

\footnotetext{
- Diálogo social y educación

* Características de los principales clientes:
}

- Tipología y número
- Participación en las ventas

- Origen de los clientes activos

* Origen y componentes de los costos:

- Origen y volumen de costos

- Tamaño y participación en las compras de los proveedores activos

* Indicadores de distribución del valor agregado:

- A empleados (i.e. promedio anual de ingresos, relación entre mayor y menor salario, distribución por características básicas)

- Al Estado (i.e. tipos de impuestos)

- A los inversionista (i.e. promedio de costos de financiación, rentabilidad, períodos),

- A la comunidad (i.e. tipos de inversión, monto, horas de trabajo voluntario, donaciones)

- A los accionistas (i.e. monto, origen, concentración, relación entre valor contable/capitalización en bolsa)

* Indicadores de reinversión en la empresa

En términos generales, estos indicadores siguen, los lineamientos propuestos por 
las guías de la Global Reporting Initiative $(\mathrm{GRI})^{4}$. Una de las ventajas más importantes de un cuarto estado financiero como el aquí descrito es que evita el divorcio entre la dimensión económica y la dimensión social. Su información es conciliable con la contabilidad tradicional, provee elementos para aumentar el impacto del desempeño social y es verificable. Según el señor Perera, se trata de un aporte a la imagen de la empresa como algo más que un ente que persigue exclusivamente el beneficio de los accionistas. Este reporte muestra a la compañía como un organismo esencial para la creación y distribución de la riqueza en las sociedades.

\section{El caso ENRON}

El descubrimiento de ingresos inflados y pasivos no registrados en ENRON causó una agitación en la economía global y en la profesión contable. ENRON, una empresa comercializadora de energía con sede en Houston, Texas, era famosa por anunciar grandes utilidades en las recientes desreguladas industrias de la electricidad y el gas natural.

El éxito de ENRON tenía un lado oscuro. No sólo era innovadora en el negocio de la comercialización de energía, sino que además, sustentaba su crecimiento en mercados emergentes mundiales sobre un considerable endeudamiento. Con la ayuda de su auditor Arthur Andersen, ENRON también infló sus ingresos de una manera indetectable para el inversionista promedio.

Es necesario considerar que siendo la séptima empresa más grande de Estados Unidos, estaba premiada por la Revista Fortune como la empresa más innovadora, más admirada y todo el mundo decía de ella que era uno de los mejores lugares para trabajar... Por supuesto que Enron se distinguía por sus balances sociales ejemplares que detallaban sus políticas anticorrupción, sus hazañas medioambientales y sus benevolentes proyectos comunitarios. Se conoce el fin de la historia, la corrupción y el fraude, la crisis bursátil, la caída de la famosa firma auditora Arthur Andersen. Uno de los tantos impactos del caso Enron, es la de haber incrementado la sospecha alrededor de los reportes de RSE.

Lo que reveló el caso ENRON es:

a. El uso de la contabilidad creativa. El hallazgo de ENRON es que no recurrió para conseguir dinero a la emisión de acciones ni al endeudamiento sino a la creación de miles de empresas en paraísos fiscales conocidas como "entidades con cometido especial", creando un conglomerado económico extraordinario pero sin balances consolidados, de tal manera que la realidad reflejada en los balances de ENRON era una parte de la realidad. No toda la realidad.

b. Las relaciones de las empresas con el poder político. ENRON efectuó grandes donaciones a las campañas de los dos partidos políticos de los EE.UU y también contribuciones a las campañas personales de los representantes.

c. El espectáculo de la clase empresarial devenida en clase política. Ha quedado a la vista que figuras prominentes de la política provienen del campo empresarial que en el pasado se han enriquecido aprovechando algunos de los hechos, circunstancias, procedimientos, instrumentos 0 herramientas que hoy son criticados por sus implicancias.

d. Las altas y desproporcionadas remuneraciones de los ejecutivos. El otorgamiento de opciones para compra de acciones.

e. La incapacidad o la complicidad de las sociedades de auditoría y de controles para detectar los fraudes o colaborar en la falsificación de las cuentas de resultados.

f. El convencimiento o el darse cuenta que se ha vivido años dentro de una burbuja financiera.

g. Los negocios que provoca el uso indebido de la información privilegiada. Hubo

4. Creado en 1997, con el respaldo del Programa de las Naciones Unidas para el medioambiente, tuvo como fin crear una guía para la elaboración de las memorias empresariales de sustentabilidad expresando los impactos económicos, sociales y ambientales de la actividad empresarial de la manera más fiable posible 
quienes se aprovecharon y vendieron sus acciones sabiendo que la debacle era inevitable.

h. La revolución que encarnó ENRON puesta en juicio. ENRON no era sólo una empresa de energía. Fue un símbolo de la gestión exitosa, nueva, revolucionaria. El elogio de la audacia sobre lo razonable y de la creatividad sobre el control. Los especialistas pusieron a ENRON como ejemplo del buen management para liderar una especie de revolución que, según algunos, es una destrucción creativa.

\section{El caso Comercial Mexicana}

Controladora Comercial Mexicana (CCM) es una empresa mexicana que nació en 1932 como una tienda de textiles en la Ciudad de México cuyo fundador fue Antonio González Abascal. En 1962 abre sus puertas la primera tienda Comercial Mexicana en la Ciudad de México. Para el año de 1970 CCM contaba con 30 tiendas distribuidas en la República Mexicana. En 1980 CCM adquiere las tiendas Sumesa, agregando así a sus tiendas de autoservicio otro formato, a la vez que abre el primer restaurante California. En el año de 1990 CCM decide abrir sus operaciones a inversiones en la Bolsa Mexicana de Valores, además hacen un acuerdo tipo "Joint venture" con Cotsco para abrir estas tiendas en México. Para el año 2000 adquieren las tiendas Auchan, se inaugura su primer centro de distribución y abre la primera tienda City Market y la primera tienda Alprecio.

En 2008, convertida en la tercera cadena de supermercados con una larga tradición entre los consumidores mexicanos, sufrió un quebranto superior a los 1,000 MDD atribuidos a pérdidas en el mercado de derivados por la negligencia de sus gestores ${ }^{5}$. Apuntar hacia la búsqueda de culpables es una tarea del consejo de administración de la propia empresa y, en todo caso, de las autoridades financieras competentes.

Lo relevante del caso de Comercial Mexicana -y de otras empresas mexicanas en situación similar- es desnudar qué falló en el sistema financiero y bursátil mexicano para que estas empresas llegaran hasta donde lo hicieron. Nadie ni nada los atajó. A modo de reflexión podemos señalar:

a) Los consejos de administración. A pesar de las nuevas leyes siguen funcionando con escaso profesionalismo y rigor. En muchos casos se impone la simulación sólo para el llenado de las formas. El papel de los consejeros independientes y, en general, de los consejeros, sigue, en muchos casos, alejado del análisis, evaluación y supervisión de las actividades de la empresa.

b) Los auditores externos y los calificadores de riesgos. Ambos se encuentran presionados y expuestos a conflictos de interés con la definición de su labor. Esto hace que buena parte de sus advertencias o resultados sean cuestionados por los inversionistas.

c) Los analistas financieros de los intermediarios bursátiles. Se requiere fortalecer la independencia y calidad de sus análisis y resultados que favorezcan la confianza de los inversionistas.

d) Los analistas de riesgos crediticios en la banca no se hicieron notar en la autorización de millonarias líneas de crédito para actividades especulativas.

f) Las leyes en el mercado de valores, si bien han sido remozadas y actualizadas para una mejor regulación y supervisión de las actividades de los mercados bursátiles en el país, su aplicación sigue siendo deficiente en: A. Oportunidad para la detección de irregularidades. B. Parcialidad en la supervisión de mercados integrados y globales. C. Mayor fortaleza institucional del supervisor, alejado de cualquier sombra que empañe el desempeño de su labor.

El quebranto de Comercial Mexicana, y de otros más, es un caso que revela los agujeros que tiene el sistema bursátil mexicano y que deben ser atendidos bajo los principios de corresponsabilidad, transparencia y globalización de mercados.

5. Negligencia de caer en la especulación, apostaron a un peso fuerte e invirtieron comprando derivados, pero el peso se devalúo ocasionado con ello un crecimiento estratosférico de su deuda. 


\section{Resumen y conclusiones}

Tomando en cuenta la Responsabilidad Social Empresarial, las finanzas incorporan formalmente los aspectos sociológicos necesarios en una dirección que apunta hacia una ciencia económica más en consonancia con las realidades y tendencias sociales actuales.

Se sabe que desde 1950 aparece la inversión socialmente responsable cuando grupos religiosos cuáqueros, decidieron dejar de invertir en actividades que dañaban la moral de la sociedad, hasta nuestros días en que se sabe que este tipo de inversión ha llegado a ser más del $10 \%$ del total en las principales bolsas de valores del mundo.

En cuanto a la creación de índices de inversiones socialmente responsables, desde 1988 se ha dado el fenómeno de la creación de estos índices con el fin que los inversionistas tengan la posibilidad de elegir invertir en aquellas empresas que se conducen dentro de los parámetros de la RSE.

Con respecto a la relación entre rendimiento económico y responsabilidad social no existe un consenso en los resultados de los estudios realizados, sin embargo, son más numerosos aquellos que han comprobado que existe una relación positiva entre la RSE y la Rentabilidad, lo que resulta un aliento para que las empresas sigan esforzándose cada vez más para alcanzar los parámetros de la RSE.

Acerca de la normatividad de la RSE en el ámbito financiero existe una amplia presencia de iniciativas que configuran el actuar de estas instituciones dentro de los parámetros de la RSE y a las que cada vez se adhieren más instituciones financieras.

Además aunque no existe un formato para presentar informes acerca de la RSE, cada vez son más las empresas que los presentan y cada vez más los inversionistas que toman en cuenta esta información al momento de decidir el destino de sus inversiones.

Los casos de Enron y Comercial Mexicana muestran la cada vez más creciente necesidad de que las empresas se conduzcan siguiendo los parámetros de la RSE, con el fin de refrendar su razón de permanecer o formar parte de una sociedad.
Es necesario considerar que la falta de imparcialidad de los auditores, a los que paga la empresa y por lo tanto sus directores, y el aumento de prestación de servicios de asesoría a las mismas empresas que están auditando, con el consiguiente interés del auditor para que se produzca una mayor rentabilidad en estas empresas. Los auditores tienen el deber de salvaguardar la inversión de los inversionistas tanto en el presente como en el futuro, pero al mismo tiempo, tienen muchos incentivos para mostrar una excesiva preocupación sólamente por el presente y por las necesidades de los gestores del negocio, en lugar de las necesidades de los inversionistas. Por lo tanto, puede ser que no sean tan independientes como se espera de ellos o como se debe entender.

La Responsabilidad Social Empresarial sólo tendrá solidez cuando logre penetrar la cultura de los actores de las organizaciones, principalmente los colaboradores internos y los usuarios directos (consumidores, clientes). Cuando la mayoría de los consumidores sean conscientes, informados y exigentes, cuando la mayoría de los empleados se formen en el tema y se comporten desde dentro como líderes vigilantes de las iniciativas, la Responsabilidad Social se volverá, ya no un lujo, una excepción, sino lo "normal", "lo que la gente hace", es decir, el Ethos común de las organizaciones. Es por eso que el factor educativo e informativo es el factor clave, y depende en gran medida a las instituciones educativas introducir el tema en la formación básica y avanzada de los estudiantes, porque son los futuros consumidores conscientes y empleados responsables de las organizaciones.

Conjuntamente con este esfuerzo cultural y educativo, los países deberán fijar reglas más exigentes para las empresas; las normas y certificadoras deberán unirse, estandarizar sus criterios y procedimientos, e internacionalizarse hasta volverse indispensables para hacer negocios.

Se debe aprender de la crisis de la última década, que tomó por sorpresa a los inversionistas, pues, debido a que los mismos no le exigían a las empresas un nivel mínimo de revelación en temas considerados críticos. Los tiempos han cambiado y ahora estarán cada vez más atentos los inversionistas por lo que será necesario que las empresas en su totalidad adopten las normas de la RSE. 


\section{Referencias}

Bolsa de Madrid (Junio, 2004) La dimensión social de las empresas y los desafíos del siglo XXI. Revista Empresas, n. 132 pp. 34-37.

Carroll, A. (July-August, 1991). The pyramid of corporate social responsibility: Toward the moral management of organizational stakeholders. Bussines Horizons.

CECA (Confederación Española de Cajas de Ahorro) (2009). Libro verde sobre la Responsabilidad Social Corporativa en el sector financiero: Una aproximación a la sostenibilidad desde las entidades financieras. España: Responsables Consulting.

Consejo Profesional de Ciencias Económicas (2010). Responsabilidad social empresaria: un enfoque financiero. Buenos Aires, Argentina: Edicon.

Corporación Andina de Fomento (2004). Finanzas sostenibles en América Latina. Unidad de publicaciones de la CAF.: Caracas, Venezuela.

De la Cuesta, M. (2005). Las inversiones socialmente responsables como palanca de cambio económico y social.Revista Futuros, III (11), consultado el 10 de junio de 2011 en http://www.revistafuturos.info/futuros_11/inversiones1.htm.

Deloitte (2011). Índice de sustentabilidad de la Bolsa Mexicana de Valores. México D.F.: Galaz, Yamazaki, Ruiz Urquiza, S.C.

Devoto, P. (2005). El concepto de responsabilidad social empresaria (RSE) frente al trabajo decente. Buenos Aires, Argentina: Organización Internacional del Trabajo, Red académica para el diálogo social.

Drucker, P. (1993). The Post capitalist Society. New York: Harper Business.

Flores, J., Ogliastri, E., Peinado-Vara, E. y Petry, I. (2007). El argumento empresarial de la RSE: 9 casos de América Latina y el Caribe. Washington D.C.: Banco Interamericano de Desarrollo-INCAE.

Ferruz, L., San Juan, I., Muñoz, F. (2009). Fondos de inversión éticos, ecológicos y socialmente responsables en Europa (1999- 2007). Aposta Revista de Ciencias Sociales, 41 (2) pp. 1-28.
Friedman, M. (September 13, 1970). The Social Responsibility of Business is to Increase Its Profits. The New York Times Magazine.

García, M. (2004). RSC. Círculo virtuoso: Responsabilidad y medioambiente. Tesis doctoral. Madrid, España: Universidad Politécnica de Madrid.

Gil, E. (2008). El éxito de la gestión ambiental en Alpina S.A. Revista - Escuela de Administración de Negocios, (62) pp. 73-87.

Gómez, E. (2007). La Responsabilidad Social Empresarial una alternativa para mejorar los niveles de vida de una sociedad y sus limitaciones. En las Memorias del XII Conferencia Anual de Ética, Economía y Dirección, Jaén 3 y 4 de junio.

KPMG (2010). Sostenibilidad. Información Integrada. Completando el ciclo estratégico. Madrid, España: KPMG.

Kramer, M. y Porter, M. (Diciembre, 2006). Estrategia y Sociedad. Harvard Business Review. América Latina.

Martínez, D. (2007). La responsabilidad social empresarial, el papel de los gobiernos, los organismos multilaterales y las ONG's. México D.F.: Universidad Anáhuac.

Núñez, G. (2003). La responsabilidad social corporativa en un marco de desarrollo sostenible en Serie Medio Ambiente y Desarrollo n. 72. Santiago de Chile, Chile: Naciones Unidas-CEPAL.

Ortiz, E. y Crowther, D. (2004) ¿Son compatibles la responsabilidad económica y la Responsabilidad Social Empresarial? IX Congreso Internacional del CLAD sobre la Reforma del Estado y de la Administración Pública. Madrid, España, 2-5 nov.

Rodríguez, A. (Febrero, 2007). La Responsabilidad Social Empresarial y las Finanzas. Revista Argentina de Ejecutivos de Finanzas.

Saavedra, M. (2008). La crisis financiera estadounidense y su impacto. Economía, (26) pp. 11-41.

Sánchez, P. (noviembre-diciembre, 2011). Índice de sustentabilidad mexicano: transparencia y sustentabilidad a largo plazo. GanarGanar, pp.18-21. 
Sen, A. (2003). Sobre ética y economía. Madrid, España: Alianza Editorial, S.A.

Vicente, A., Ruiz, M.,Tamayo, U. y Balderas, A. (2004). Compatibilidad entre responsabilidad social corporativa y competitividad: Estado de la cuestión en el ámbito Internacional. Bizkaia, España: Instituto de Eco- nomía Aplicada a la Empresa,Universidad del País Vasco:

Vives, A. y Peinado, E. (2004). La Responsabilidad Social de la empresa del dicho al hecho. Anales del II Conferencia Interamericana de Responsabilidad Social en la empresa, Ciudad de México, 26,27 y 28 de septiembre. 